\title{
Cutaneous Findings of COVID-19: A Review of the Literature
}

\author{
(1) Mahmut Can Koska, (1) Hülya Süslü²
}

${ }^{1}$ Artvin Public Hospital, Clinic of Dermatology, Artvin, Turkey

2University of Health Sciences Turkey, Haseki Training and Research Hospital, Clinic of Dermatology, Istanbul, Turkey

*Each author contributed to this article equally

\section{ABSTRACT}

Many different cutaneous findings have been reported in coronavirus disease 2019 (COVID-19) patients. It is still uncertain whether these findings are associated with disease. On the other hand, lesions had different features, time of onset and prognostic relations. We reviewed published data in PubMed database with keywords of "COVID-19" and "cutaneous". We found out 34 articles consisted with 563 patients. Urticarial rash was the most common followed by chilblain-like and vesicular lesions. However, total number non-specified maculopapular rashes were higher than other lesions according to one article which included both confirmed and suspected patients. Livedo-like lesions and acro-ischemia tent to appear in severe COVID-19 patients. Chilblain-like lesions were reported more frequently in young patients at late periods of disease and also, in young normal population without history of COVID-19. Petechial and purpuric lesions were developed either true vasculitis or thrombogenic vasculopathy. Vesicular eruptions resembled to herpes simplex virus and varicella-zoster virus infections and these infections should strongly be considered. More studies and reports are needed to determine non-specific maculopapular and rare lesions such as mottling. Although many reports and classifications exist about cutaneous findings of COVID-19, their exact relationships remain to be elucidated especially for maculopapular and urticarial lesions which can also be seen in other viral exanthems and drug eruptions. Furthermore, clinical data and histopathologic features weren't reported in several articles. In conclusion, varied types of cutaneous lesions can be seen in COVID-19 and beneficial for suspicion of disease and prognosis.

Keywords: COVID-19, Cutaneous, Skin, Dermatology, Livedo-like lesions, Chilblain-like lesions, Maculopapular, Urticaria, Vesicular rash

\section{Introduction}

Since it was first reported in China on December 2019, coronavirus disease 2019 (COVID-19) has spread throughout the world rapidly [1]. Consequences were terrible and dreadful as it causes more than 250,000 deceased people in more than 180 countries worldwide [1]. World Health Organization (WHO) declared this out-brake as pandemic and governments took precautions which resulted huge impacts on socio-economic status of communities [1,2]. Suspected virus was described by WHO as Severe Acute Respiratory syndrome Coronavirus-2 (SARS-COV-2) that belongs to family of Coronaviridae, the same family of SARS and MERS out-brakes' agents [1,3]. It is single stranded RNA virus with envelope and transmits by inhaling of expelled droplets from patients by coughing and sneezing. Contact and carry of droplets to respiratory mucosa and conjunctiva is another important transmission path. Virus uses angiotensinconverting enzyme 2 (ACE-2) to hold on and invade respiratory epithelium [1]. Median incubation period is 5.2 (4-14) days. In most cases disease begins with fever, cough, fatigue in whom dyspnea, hypoxemia may accompany. Mortality is usually resulted by respiratory failure after 6-41 days from beginning of symptoms [4]. Diagnosis is confirmed by reverse-transcriptase polymerase chain reaction (PCR) test [1]. Although respiratory system is mostly involved, other organs can also be affected especially gastrointestinal tract $[1,4]$. It is not surprising because ACE-2 is expressed in many organs, particularly in small intestines [1]. The 
first report about skin findings is from China in which prevalence was reported as $0.2 \%$ [5]. Since then many cases with varied types of skin lesions were published however, it is not certain whether these findings were associated with disease [5,6]. Some of these are relatively specific to COVID-19 such as varicella like lesions on the other hand, maculopapular rash and urticarial rash are not and can be seen in other viral infections [6]. Lesions may appear before other symptoms and recognition is essential in such cases for early diagnosis and precautions $[7,8]$. It was also speculated that several of these lesions may predict complications and poor outcome [9]. Furthermore, not all articles presented photographs of patients and described pathological features [5]. In this paper we aimed to review reported skin lesions of COVID-19 confirmed patients in whom these lesions related to disease. We also intent to categorize these and search for existence of photographs and histopathologic investigation.

We searched for articles about skin findings of COVID-19 infection in PubMed database on May 27th, 2019. We use keyword combination of "COVID-19" and "cutaneous". All accessible articles that were written in English were evaluated. We reviewed articles that reported patients with confirmed COVID-19 infection and associated skin lesions. Articles that were reviews, not relevant with skin findings of COVID-19, written in other than English, with nonconfirmed COVID-19 patients, reported skin findings not related with COVID-19 were excluded. Skin lesions that was evaluated by tele-dermatology were excluded too. We extracted demographic, clinical, histopathological data of patients and categorized them according to type of skin findings that were described by Wollina et al. [5]. Onset time of skin lesions was measured from beginning of respiratory signs of COVID-19 infection. If onset time of lesions was pointed according to hospital admission or COVID-19 diagnosis, it was also pointed in review. We also pointed existence of photographs.

\section{Review of Cutaneous Findings of COVID-19}

Searching of database reveled 85 articles. After evaluation 34 articles fulfilled the criteria and were included to the review. Six of them were studies and large case series, others were small case series and reports. Frequencies, which were reported in studies and large case series, were showed in Table 1. Characteristics of reported patients were shown in Table 2. Erythematous rash evaluated as different group due to several reports in which non-specific erythematous lesions were reported under this nomenclature.

\section{Maculopapular Rash}

In our review we reached seven articles about maculopapular rash which consisted of 14 patients. Eight females and six males were reported. Female patients' mean age was 63.2 (range: $32-84$ ) and males was 51.6 (6-88). Onset time of rash varied between 2 and 33 days from COVID-19 symptoms. Pictures of almost all lesions existed in papers $[10,11,12,13,14,15,16]$. Herrero-Moyano et al. [16] performed histopathological examination in 8 patients and observed varied findings. These were spongiosis, non-follicular sub-corneal pustules, neutrophilic interstitial infiltrates and exocytosis, rare eosinophils. They reported signs of vascular injury, microthrombi in capillaries, erythrocytes extravasation in several patients. They excluded existence of infectious agents [16]. Duration of lesions were varied between $4-16$ days $[10,11,12,13,14,15]$. Avellana Moreno et al. [14] reported patient treated with intravenous corticosteroid and antihistamines whom lesions resolved in five days on the other hand, Morey-Olive et al. [13] reported lesions that healed in five days without treatment.

\section{Erythematous Rash}

Erythematous rash were reported by seven articles in our screen. Fifty-eight patients were reported in these papers. Age and gender data were missing in some patients $[17,18,19,20,21,22,23]$. De Giorgi et al. [22] reported frequency in their series as 70 percent (37 patients over 53). Most of these patients had mild itch. In one report 39-yearold female developed non-pruritic, annular fixed, erythematous and edematous plaques that located on upper extremities, chest, abdomen, neck and palms. Rash resolved in seven days and no therapy was reported in that patient [18].

\section{Pityriasis Rosea Like Eruption}

We found only two reports about Pityriasis Rosea like eruption. Galvan Casas et al. [24] reported 47 patients over 375 but, no clinical features were reported. Ehsani et al. [25] reported 27-year-old man developed erythematous and scaly annular plaques. These located on forearm, trunk and upper extremities and scattered like pinetree branches. Rash had appeared three days after from low grade fever and non-specific symptoms and continued to appear five

Table 1. Reported frequencies of cutaneous findings in COVID-19 patients from studies and case series

\begin{tabular}{|l|l|}
\hline Author & $\begin{array}{l}\text { Frequency of skin findings in } \\
\text { COVID-19 (reported from studies } \\
\text { and case series) } \\
\text { Number of patients and percentages }\end{array}$ \\
\hline Fernandez-Nieto et al. [40] & $24 / 53$ (NR) \\
\hline Hedou et al. [20] & $5 / 103($ NR) \\
\hline Recalcati [21] & $18 / 88(20.4 \%)$ \\
\hline De Giorgi et al. [22] & $53 / 678(7.8 \%)$ \\
\hline Guarneri et al. [23] & $13 / 125(10.4 \%)$ \\
\hline Herrero-Moyano et al. [16] & $8 / 1177(0.7 \%)$ \\
\hline NR: Non-reported, COVID-19: Coronavirus disease 2019 \\
\hline
\end{tabular}




\begin{tabular}{|c|c|c|c|c|c|c|}
\hline 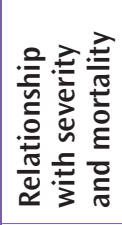 & $\frac{o v}{z}$ & 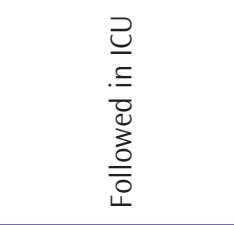 & $\frac{\text { zu }}{z}$ & $\stackrel{o}{z}$ & $\frac{o v}{z}$ & $\frac{o}{z}$ \\
\hline 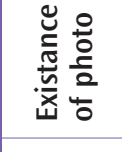 & 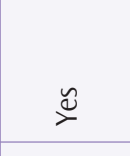 & $\stackrel{\check{\varrho}}{=}$ & $\frac{\tilde{z}}{z}$ & $\stackrel{o}{z}$ & $\frac{o}{z}$ & $\stackrel{\check{c}}{=}$ \\
\hline 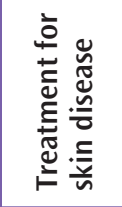 & 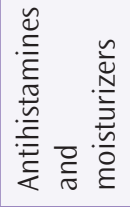 & $\frac{o}{z}$ & $\frac{o v}{z}$ & 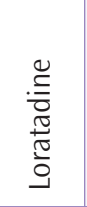 & 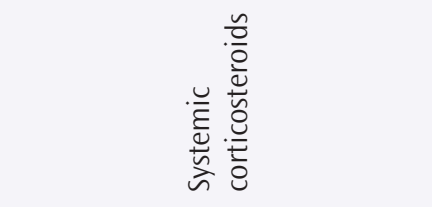 & $\frac{o u}{z}$ \\
\hline 高高 & $\frac{8}{z}$ & $\stackrel{\Xi}{=}$ & z & $\frac{\alpha}{z}$ & 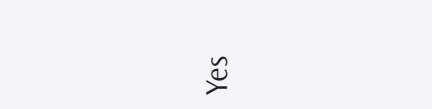 & $\stackrel{ \pm}{N}$ \\
\hline 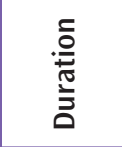 & $\begin{array}{l}\text { 管 } \\
\text { N }\end{array}$ & 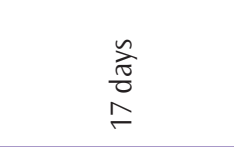 & $\frac{o}{z}$ & $\underset{\leftarrow}{\frac{\pi}{6}}$ & 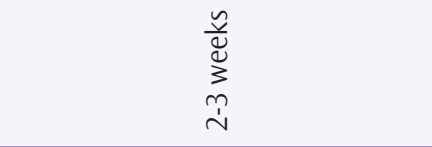 & 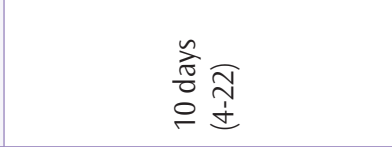 \\
\hline 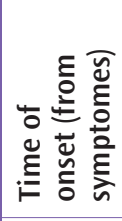 & 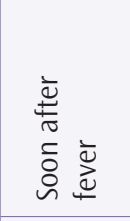 & 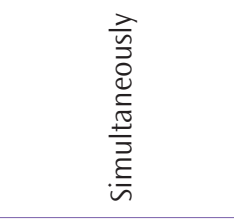 & 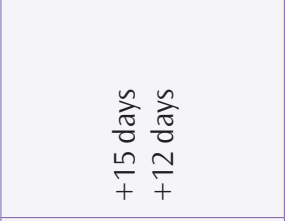 & 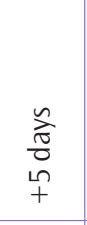 & 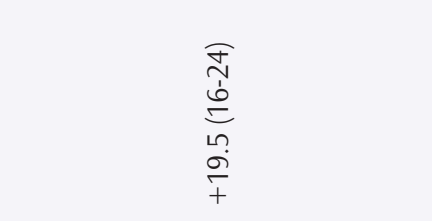 & 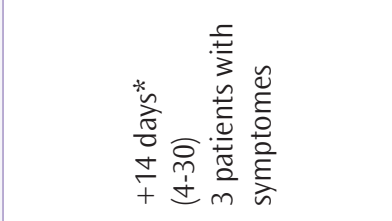 \\
\hline 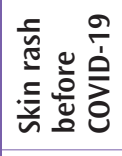 & $\stackrel{\circ}{z}$ & $\stackrel{0}{z}$ & 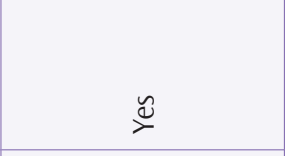 & z & $\stackrel{\circ}{z}$ & 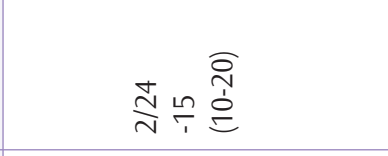 \\
\hline 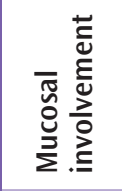 & zo & ż & z & 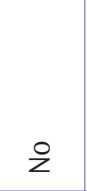 & 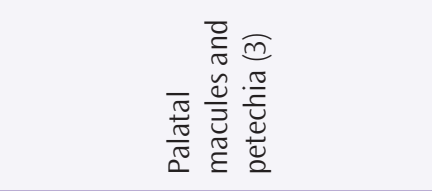 & ż \\
\hline 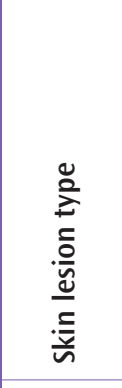 & 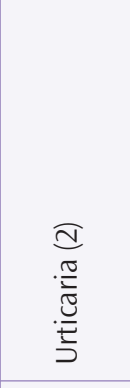 & 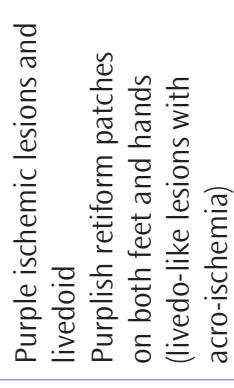 & 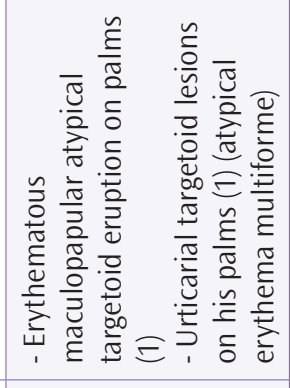 & 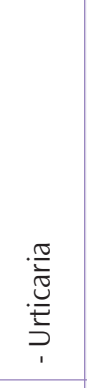 & 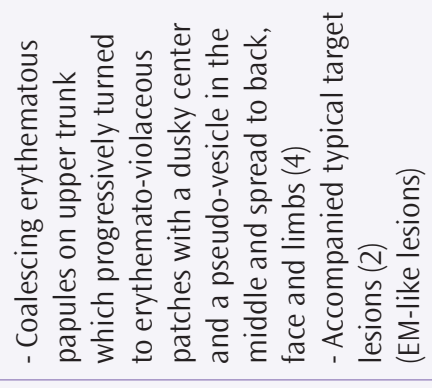 & 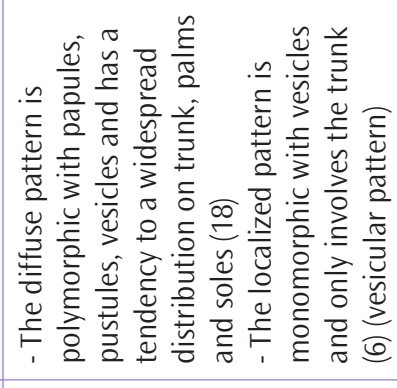 \\
\hline 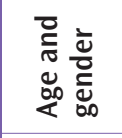 & 㟔峁 & $\sum_{\sigma}$ & 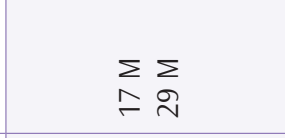 & $\sum_{\Sigma}$ & 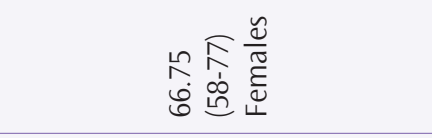 & 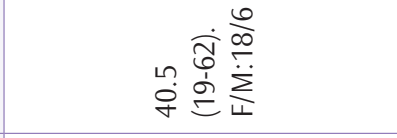 \\
\hline 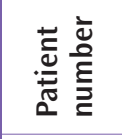 & $N$ & - & N & - & $\sigma$ & \pm \\
\hline 高 & 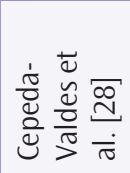 & 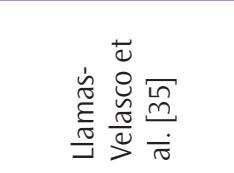 & 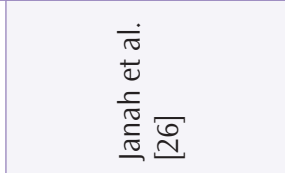 & 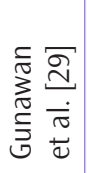 & 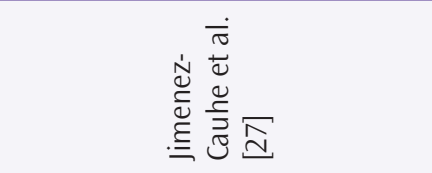 & 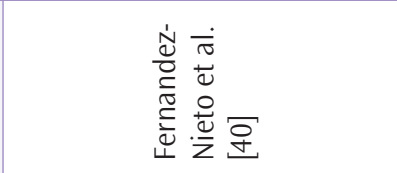 \\
\hline
\end{tabular}




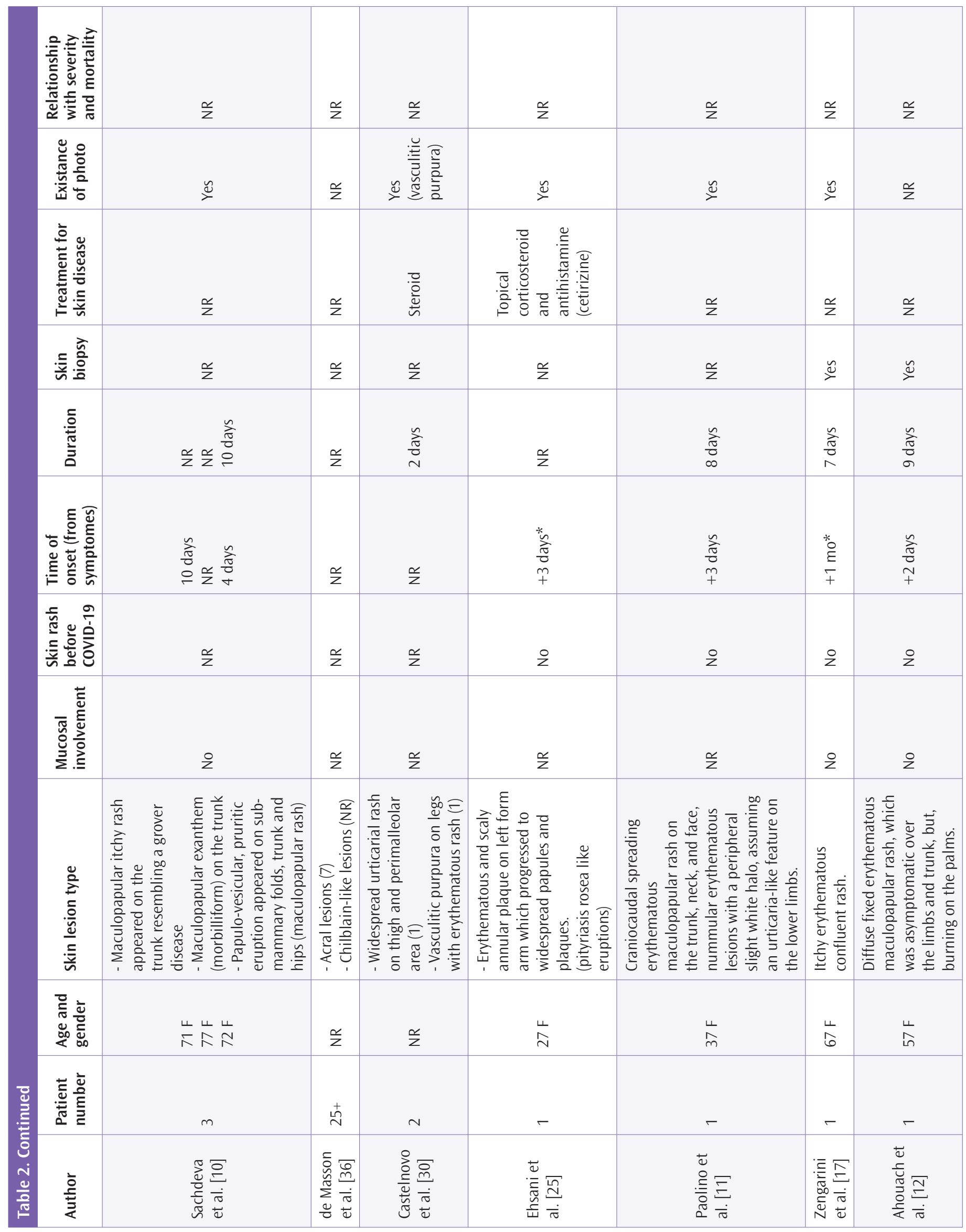




\begin{tabular}{|c|c|c|c|c|c|}
\hline 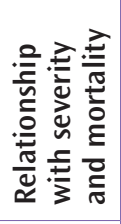 & 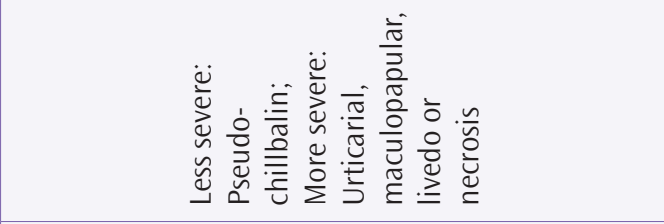 & $\frac{o}{z}$ & $\frac{o}{z}$ & $\frac{o}{z}$ & $\frac{o}{z}$ \\
\hline 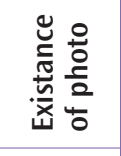 & 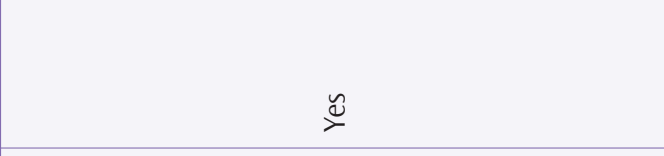 & $\check{\widetilde{0}}$ & $\stackrel{o}{z}$ & $\check{\check{\Sigma}}$ & $\check{\check{\nu}}$ \\
\hline 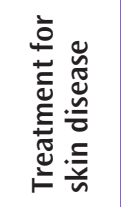 & $\frac{o x}{z}$ & 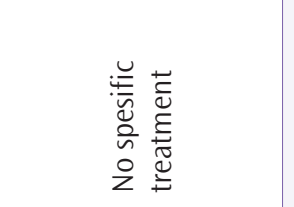 & $\stackrel{o}{z}$ & 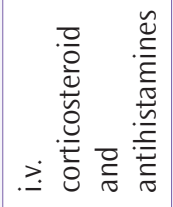 & $\frac{o}{z}$ \\
\hline 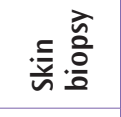 & $\frac{\sim}{z}$ & $\frac{\alpha}{z}$ & $\frac{o}{z}$ & $\frac{\infty}{z}$ & $\check{\check{x}}$ \\
\hline 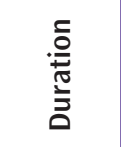 & 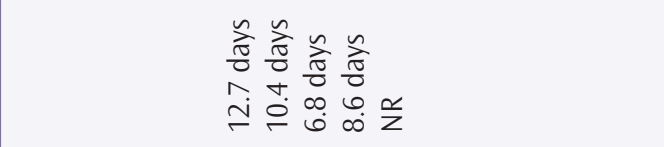 & $\begin{array}{l}\frac{n}{\tilde{E}} \\
\text { in }\end{array}$ & $\frac{\alpha}{z}$ & $\begin{array}{l}\frac{\tilde{u}}{\tilde{J}} \\
\frac{\sigma}{\sigma}\end{array}$ & $\begin{array}{l}\frac{n}{\tilde{\delta}} \\
\frac{\delta}{n}\end{array}$ \\
\hline 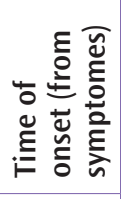 & $\frac{o}{z}$ & 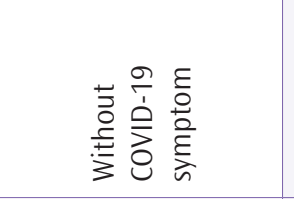 & 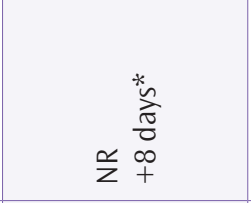 & 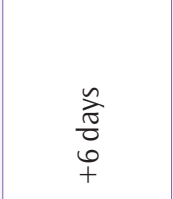 & 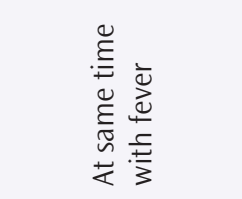 \\
\hline 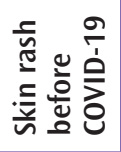 & 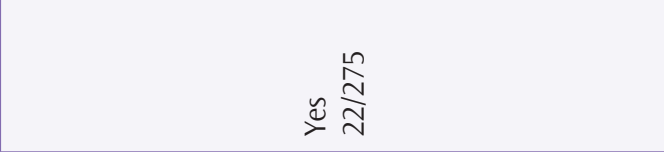 & 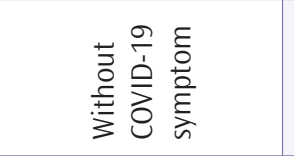 & $\frac{o}{z}$ & $\stackrel{2}{z}$ & $\stackrel{2}{z}$ \\
\hline 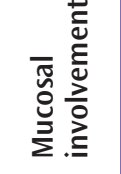 & $\frac{\alpha}{z}$ & $\stackrel{\gtrless}{2}$ & $\frac{\alpha}{z}$ & 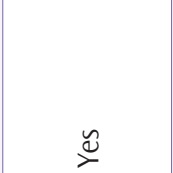 & $\stackrel{2}{z}$ \\
\hline 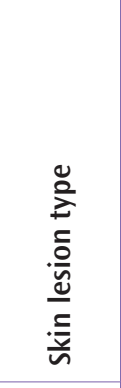 & 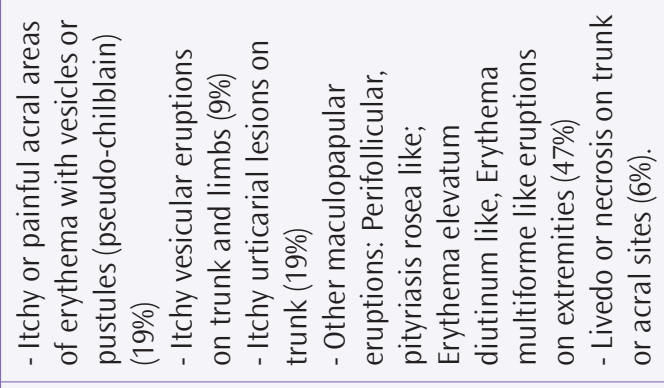 & 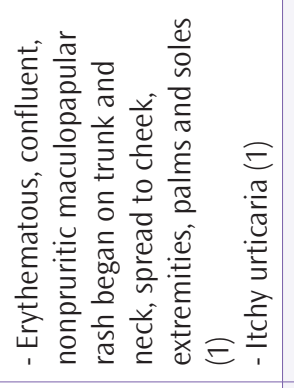 & 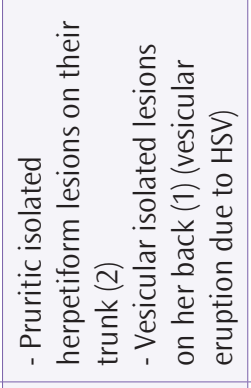 & 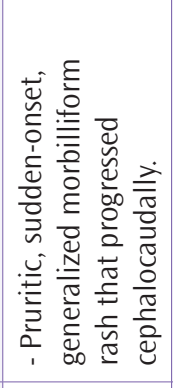 & 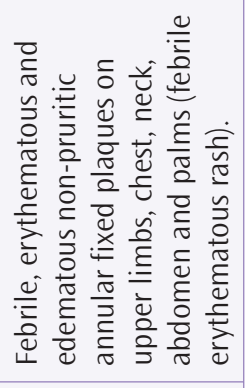 \\
\hline 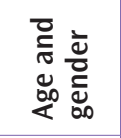 & $\frac{o}{z}$ & 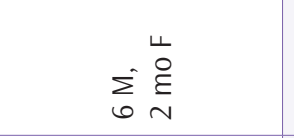 & $\frac{o}{z}$ & $\underset{\sim}{\stackrel{u}{m}}$ & 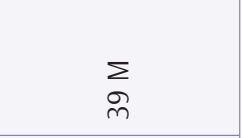 \\
\hline 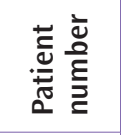 & 点 & N & $m$ & - & - \\
\hline 嵩 & 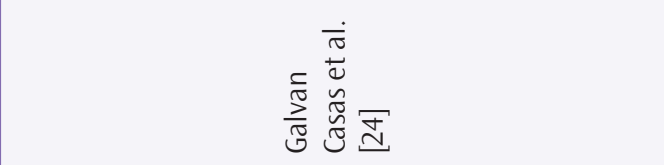 & 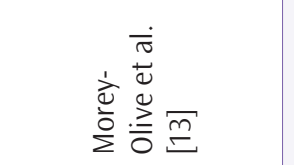 & 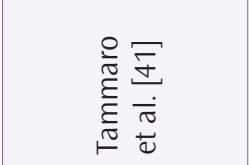 & 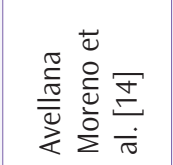 & 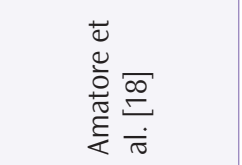 \\
\hline
\end{tabular}




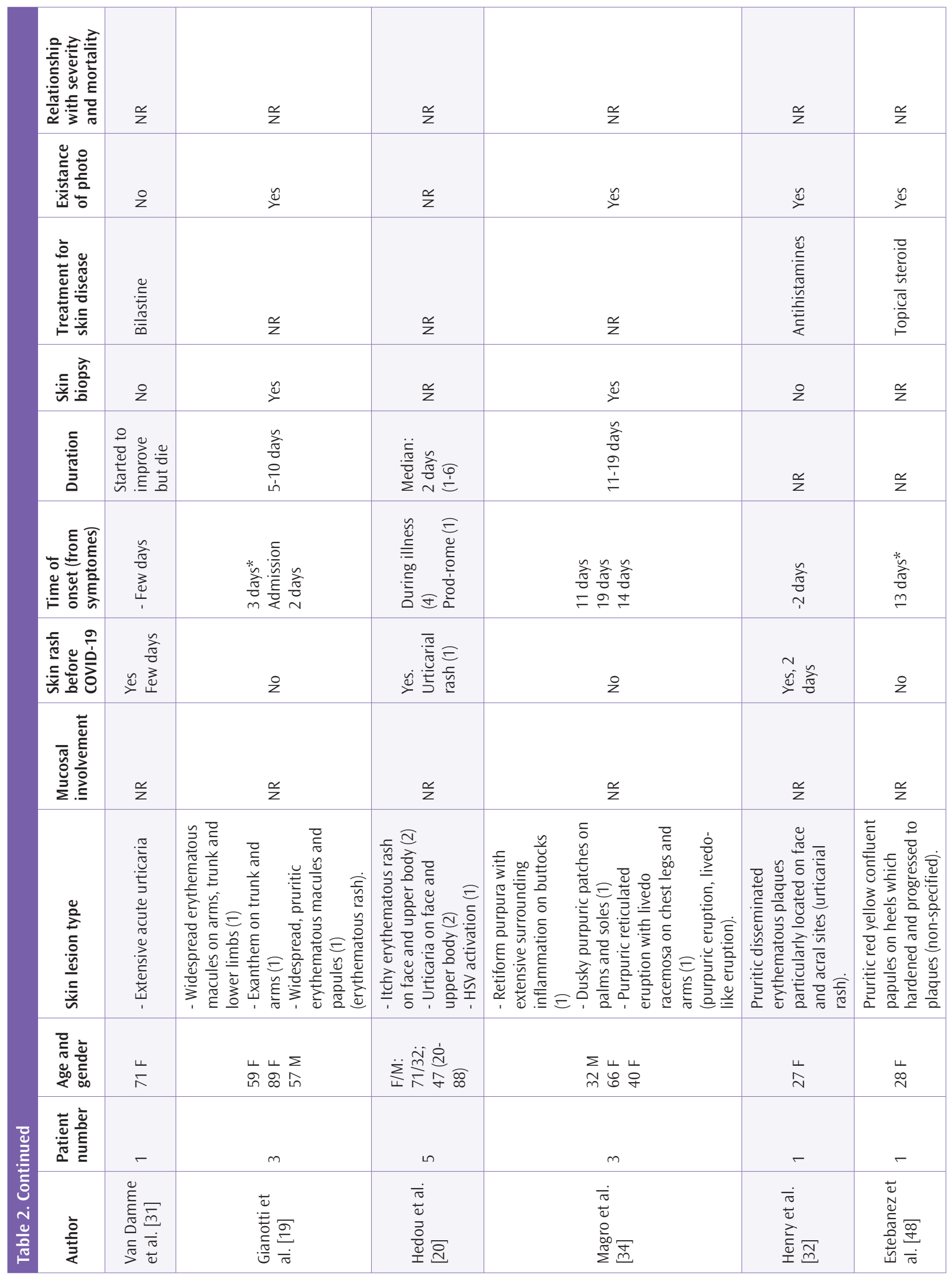




\begin{tabular}{|c|c|c|c|c|c|c|c|}
\hline 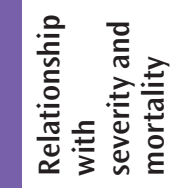 & $\stackrel{o}{z}$ & $\stackrel{o}{z}$ & $\stackrel{o}{z}$ & $\stackrel{\sim}{z}$ & $\stackrel{o}{z}$ & $\stackrel{o}{z}$ & $\stackrel{o}{z}$ \\
\hline 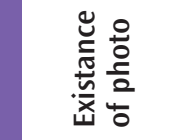 & $\stackrel{\varpi}{\check{\nu}}$ & $\frac{\alpha}{z}$ & $\stackrel{\varrho}{z}$ & 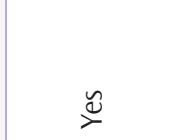 & $\stackrel{c}{z}$ & 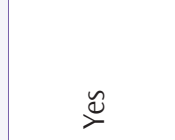 & $\stackrel{c}{z}$ \\
\hline 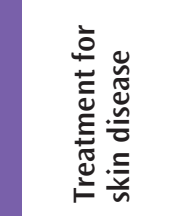 & 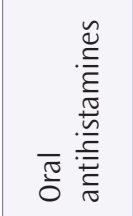 & $\stackrel{o}{z}$ & 兰 & 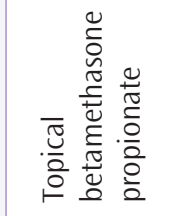 & $\stackrel{\varrho}{z}$ & 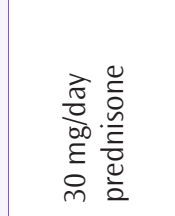 & $\stackrel{\varrho}{z}$ \\
\hline 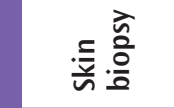 & $\stackrel{\check{\nu}}{\check{\nu}}$ & $\stackrel{o}{z}$ & $\stackrel{o}{z}$ & 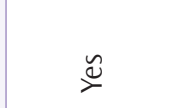 & $\frac{\alpha}{z}$ & 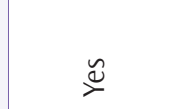 & $\stackrel{o}{z}$ \\
\hline$\frac{\bar{\sigma}}{\stackrel{\frac{0}{\pi}}{\frac{5}{2}}}$ & $\begin{array}{l}\frac{n}{\hat{\sigma}} \\
\frac{\pi}{0} \\
\text { in }\end{array}$ & $\begin{array}{l}\text { ָे } \\
\text { i } \\
\text { v }\end{array}$ & 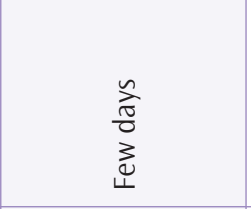 & 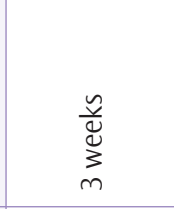 & 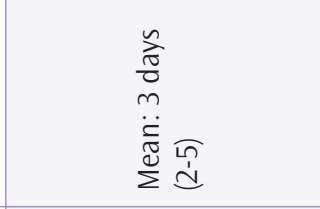 & $\begin{array}{l}\frac{n}{\pi} \\
\frac{\pi}{0} \\
?\end{array}$ & 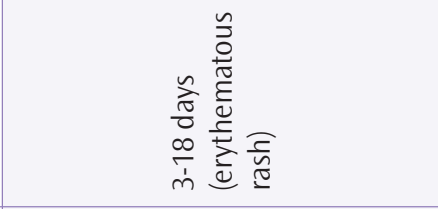 \\
\hline 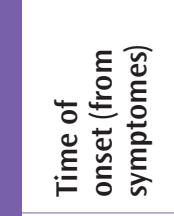 & $\frac{n}{\frac{n}{0}}$ & $\frac{\bar{\pi}}{\frac{\pi}{\frac{\pi}{x}}} \cdot \frac{\frac{\sigma}{\tilde{n}}}{\frac{0}{\sigma}}$ & 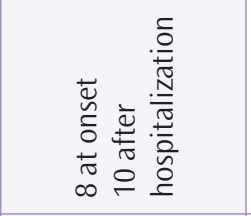 & $\underset{\substack{\frac{\pi}{0} \\
r}}{n}$ & 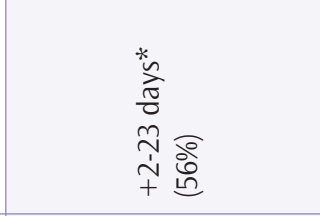 & $\begin{array}{l}\stackrel{\wp}{\xi} \\
\mp\end{array}$ & 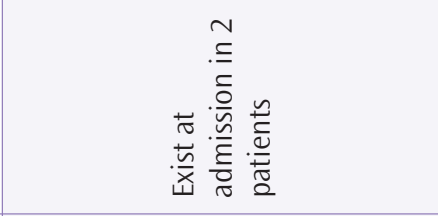 \\
\hline 畜 $\frac{0}{\grave{\omega}}$ & 우 & 2o & $\stackrel{o}{z}$ & ì & 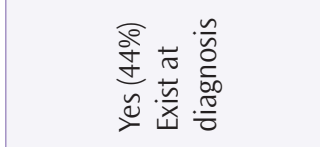 & $\stackrel{\circ}{z}$ & $\stackrel{o}{z}$ \\
\hline 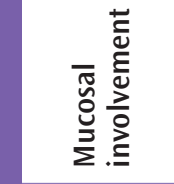 & $\frac{\alpha}{z}$ & $\stackrel{\mathscr{c}}{z}$ & $\frac{\alpha}{z}$ & z & $\stackrel{o}{z}$ & $\frac{a}{z}$ & $\stackrel{\varrho}{z}$ \\
\hline 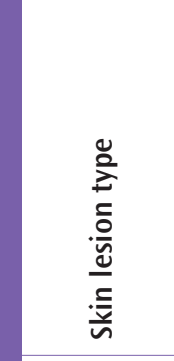 & 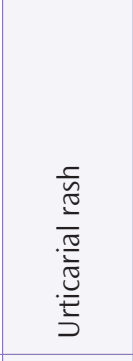 & 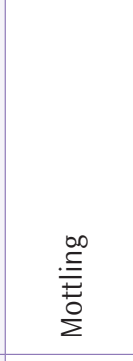 & 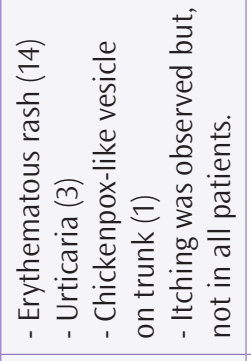 & 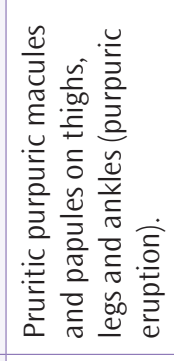 & 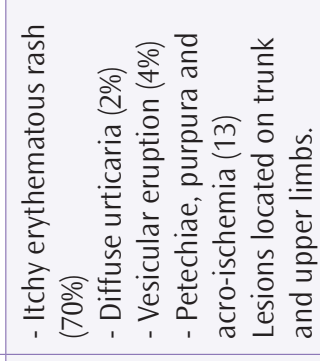 & 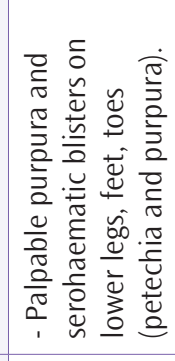 & 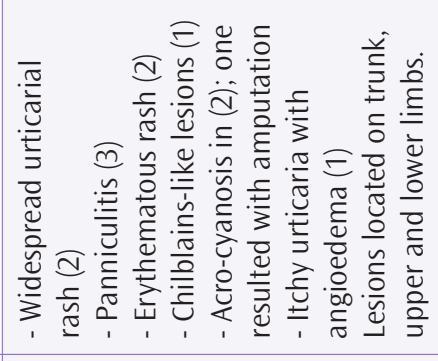 \\
\hline 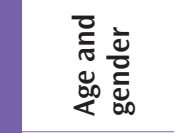 & $\stackrel{\vec{m}}{\stackrel{m}{m}}$ & $\stackrel{\sum}{\llcorner}$ & $\frac{\alpha}{z}$ & $\frac{L}{\Sigma}$ & 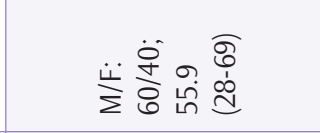 & $\underset{\infty}{\sim}$ & $\frac{\alpha}{z}$ \\
\hline 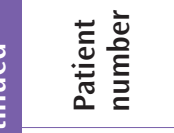 & - & - & $\stackrel{\infty}{\leftarrow}$ & - & $\hat{ก}$ & - & $\stackrel{m}{\sim}$ \\
\hline 高 & 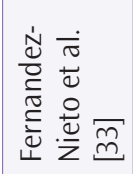 & 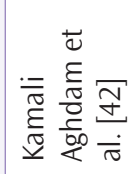 & 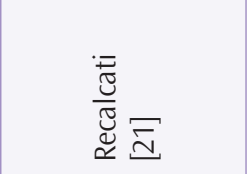 & 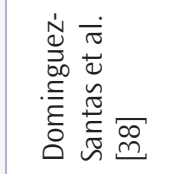 & 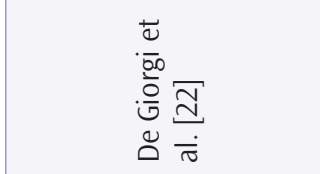 & 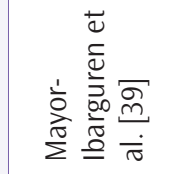 & 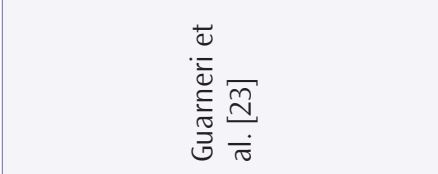 \\
\hline
\end{tabular}




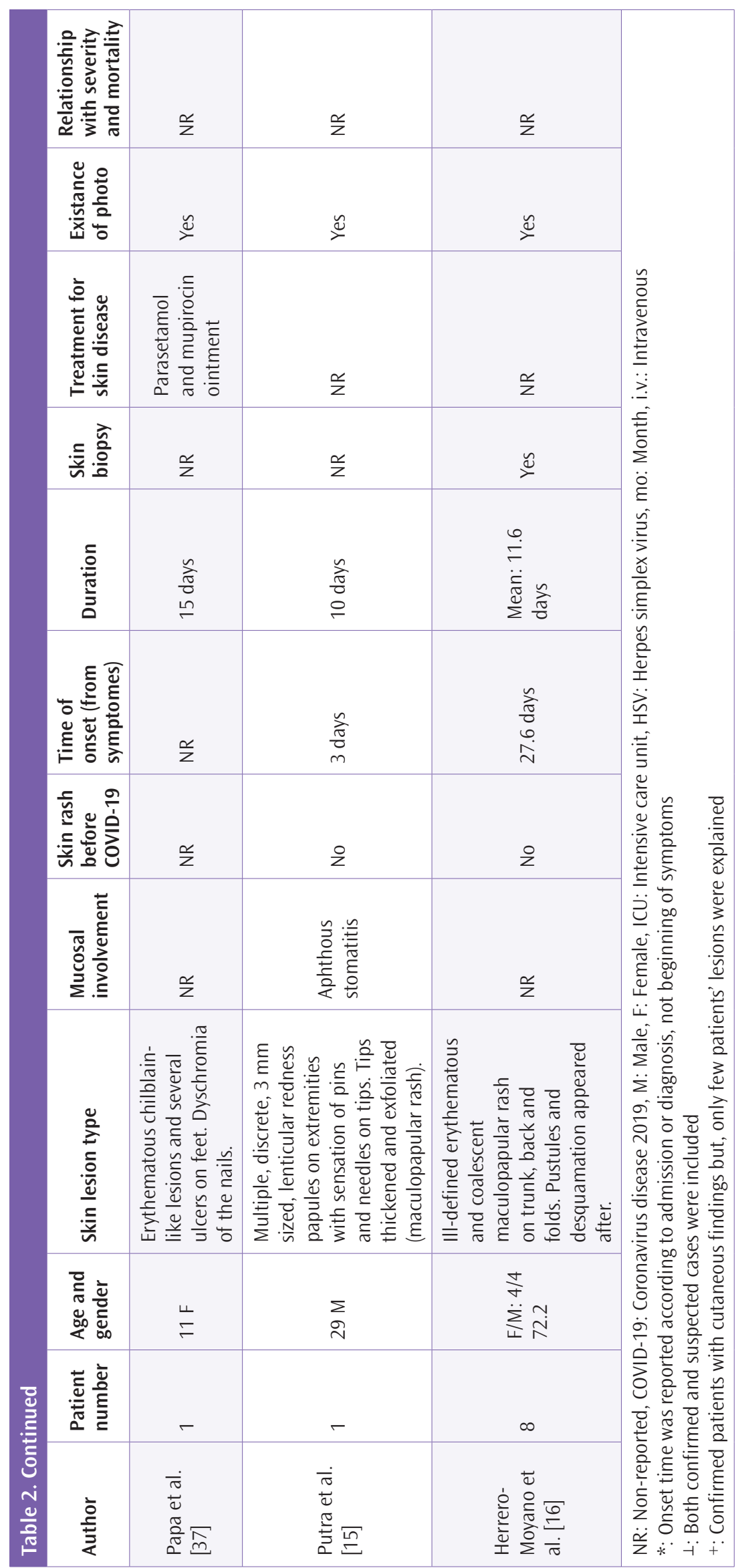

days, became pruritic and disseminated. Patient were treated with topical steroids and cetirizine.

\section{Erythema-multiforme Like Lesions}

Three articles reported erythema-multiforme like lesions. Janah et al. [26] reported two patients with ages of 17 and 29. Both developed atypical targetoid lesions on palmar regions. No mucosal lesions and history of recurrent herpes virus infection was reported. Rash appeared after 12 and 15 days from COVID-19 symptoms. Photographs existed in papers but histopathological features didn't. Jimenez-Cauhe et al. [27] reported four patients with varied ages between 58-77. They developed rash after 16-24 days from COVID-19. However, in three of them lesions appeared after discharging from clinic. All patients had lesions on face, trunk, extremities but not on palmoplantar regions. Mucosal lesions as macules and petechia, especially on palatal sites, were reported in three patients. Histopathological features were similar in all patients. These were basket-weave stratum corneum, mild-moderate spongiosis, dilated vessels filled with neutrophils, extravasation of red blood cells, perivascular and interstitial lymphocytes. Basal vacuolar changes and lymphocytic exocytosis were observed in each different patient. All patients' lesions subsequently resolved in 2-3 weeks with systemic corticosteroids. Galvan Casas et al. [24] also reported erythema-multiforme like lesions in their series consisted of 375 patients but, however, clinical data were lack.

\section{Urticarial Rash}

Urticarial lesions were reported in 104 patients by 11 articles that consisted of five case series and six case reports. One patient developed urticarial rash accompanied with angioedema. These patients were included 79 confirmed and 24 suspected cases [13,21, $22,23,24,28,29,30,31,32,33]$. Age and gender data were available in several articles. In these articles urticarial rash tent to be more frequent in females and ages were varied between two months and 71 years $[13,31]$. Development time of lesions were highly varied in reports. In series of Galvan Casas et al. [24] 73 patients with COVID-19 and urticarial rash were reported. In this series three patients developed lesions before and 43 at the same time with COVID-19 symptoms, 25 did after that. Henry et al. [32] reported lesions appeared before 
two days, on the other hand, in case report of Fernandez-Nieto et al. [33] after six days from COVID-19 symptoms. Furthermore, MoreyOlive et al. [13] reported two-month-old infant with only fever and urticarial lesions in absence of other COVID-19 symptoms. Mean time of duration of symptoms was reported 6.8 days [standard deviation (SD): 7.8] by Galvan Casas et al. [24], seven of them were treated with systemic corticosteroid. Gunawan et al. [29] reported duration time as one day. Histopathologic investigation revealed upper dermal edema, perivascular lymphocytic infiltrates and some eosinophils [33]. There was lack of data about relation between prognosis and rash.

\section{Vascular Lesions}

Many lesions that concerning vascular involvement have been reported in COVID-19 patients. These are livedo-like lesions, chilblainlike lesions, ischemic ulcers, petechia, purpura and necrotic lesions [5]. Histopathological investigations of several COVID-19 patients revealed vascular injury with or without vasculitis, microthrombi and also complement system activation and deposition in both lesions and normal skin [34].

Few varied reports exist about livedo-like, ischemic and necrotic lesions on acral sites. Livedo-like lesions consist of livedo racemosa, purple round or reticular patches located on trunk, thigh, legs, arms feet and hands [24,34,35]. Accompanied acral ischemia and retiform purpura were also reported [34,35]. Skin symptoms varied in reports from asymptomatic to painful, burning and itchy

Table 3. Types and patient numbers of cutaneous findings

\begin{tabular}{|l|l|}
\hline Type of cutaneous finding & Number \\
\hline Total patient number & 563 \\
\hline Urticarial rash & 104 \\
\hline Maculopapular rash & 14 \\
\hline Erythematous rash & 59 \\
\hline Vesicular lesions & 64 \\
\hline Petechiae, purpura, acroischemia, livedo, necrosis & 43 \\
\hline Chilblains-like lesions & 73 \\
\hline $\begin{array}{l}\text { Perifollicular; pityriasis rosea like; erythema elevatum } \\
\text { diutinum like, erythema multiforme like eruptions }\end{array}$ & 183 \\
\hline Acral lesions & 7 \\
\hline HSV activation & 3 \\
\hline Panniculitis & 3 \\
\hline Grover disease-like lesions & 1 \\
\hline $\begin{array}{l}\text { Pruritic red yellow confluent papules on heels (non- } \\
\text { specified) }\end{array}$ & 1 \\
\hline Chickenpox-like vesicle on trunk & 1 \\
\hline Mottling & 1 \\
\hline Hsv: Herpes simplex virus & \\
\hline
\end{tabular}

[24,34,35]. In a study of Galvan Casas et al. [24], which included both confirmed and suspected COVID-19 patients, together these and acro-ischemic lesions were reported in 29 patients (6\% percent). This group patients showed higher mortality on the other hand, patients that showed transient lesions experienced mild disease. In that study mean age of these patients were 63.1 with SD of 17.3 and 10 of them were females (48\%). Also confirmed cases were much more compared to suspected ones (81\% vs. 19\%). Eighteen of them (86\%) developed lesions at the same time with other symptoms, 1 before, 2 after and in 8 data wasn't reported. Mean time of duration of lesions 9.4 days with SD of 5.4. LlamasVelasco et al. [35] reported 61-year-old male patient with livedolike lesions on hands and accompanied acro-ischemia which appeared at the same time with COVID-19 symptoms. Followed in intensive care unit (ICU), lesions showed some improvement after 19 days. Magro et al. [34] reported three patients with skin lesions concerning vascular involvement. One of them, 40-year-old female developed retiform purpura and livedo racemosa on chest, legs and arms. Lesions noted at admission and she had had symptoms for 14 days. Exact time of appearance and duration of lesions and progress of disease weren't reported but, d-dimer and INR levels were elevated. Histopathological features were reported in only two reported cases. Magro et al. [34] reported in their patient perivascular lymphocytic infiltrate and microthrombi without vasculitis in venules of the deep dermis. They also investigated patients for complement activation and observed vascular C5b-9 and C4 deposition in both lesion and normal skin biopsy that obtained from deltoid area. Llamas-Velasco et al. [35] also observed deep-dermis seated thrombi but, in larger arterial vessels that were surrounded with limited neutrophils and showed focal fibrinoid necrosis. Additionally, dilated vessels that were filled with thrombi and surrounded by mild neutrophilic components were seen in papillary dermis. Upper dermis and eccrine sweat gland necrosis, particularly in secretory parts, were also accompanied.

Acro-ischemic changes without livedo-like lesions were also reported. These lesions consisted of finger and toe cyanosis [23]. Aforementioned study from Spain included acro-ischemic lesions without livedo into livedo/necrosis group and as mentioned before, reported frequency was six percent [24]. Guarneri et al. [23] reported two patients with leg thrombosis. One of them experienced amputation due to thrombosis. De Giorgi et al. [22] reported 13 patients with petechia, purpura, acro-ischemia. These group consisted of more severe patients several of whom were cared in ICU and had clotting disorders.

Chilblain-like lesions consisted of erythematous and edematous lesions which located on acral sites. Accompanied vesicles or pustules were also reported. Skin symptoms of itch, pain or burning can be seen. Galvan Casas et al. [24] reported 71 patients 
(19\%) in their series. Mean age of these patients was 32.5 (SD: 21.8) and 48 of them were females (68\%). Most of them developed the lesions after COVID-19 symptoms (42 patients, 59\%). Twentyfour patients (34\%) developed lesions at the same time of disease symptoms and 5 patients (7\%) before. Mean duration of lesions were 12.7 days and more frequently seen in mild patients. Fortytwo of these patients (59\%) weren't confirmed by virologic tests. Guarneri et al. [23] reported 1 confirmed patient with chilblainlike lesion but, clinical features weren't pointed. On the other hand, they did research with tele-dermatology for chilblain-like lesions. They found 22 patients who were scanned for COVID-19 with rhino-pharyngeal swab samples. Mean age was 14.3 with a range of 6-30 and 19 were children. COVID-19 diagnosis was confirmed in 6 of them of whom 5 were children. de Masson et al. [36] reported several cases in their series. Papa et al. [37] reported 11-year-old girl with chilblain-like lesions with erythema and several ulcers. They also observed dyschromia on nails. There was no sign of any disease in her medical history. SARS-COV-2 wasn't detected in nasopharyngeal swab but, IgG antibodies against virus was. Lesions completely resolved with topical antibiotics and analgesic in 15 days.

\section{Petechia and Purpuric Rash}

Reported petechial and purpuric rashes could be induced by either vasculitis or vascular occlusion without inflammation [34,38]. It consisted of palpable purpura, dusky purpuric patches or retiform purpura surrounded by inflammation located on lower extremities and palms and soles $[30,34,38,39]$. Pruritus was reported in several patients and Koebner phenomenon in one [30,38]. De Giorgi et al. [22] reported 13 patients with petechia, purpura and acro-ischemia over 53 COVID-19 patients which was pointed above. These patients were more severe and association with coagulation disorders was observed. Magro et al. [34] reported two patients; 32-year-old male with retiform purpura with surrounding inflammation located on thighs and 66-year-old female with dusky purpuric patches located on palms and soles. Lesions appeared after 11 and 19 days from COVID-19 symptoms, respectively. Castelnovo et al. [30] reported one patient with itching purpura on legs which suggested vasculitis. Erythematous rash accompanied to lesions. This patient developed severe respiratory failure. Lesions resolved in few days with steroid treatment. Dominguez-Santas et al. [38] reported 71-year-old man with pruritic purpuric macules and papules on both legs which extended from ankle to thighs. Koebner phenomenon was positive. Lesions appeared seventh day of COVID-19 symptoms and resolved in three weeks with topical steroid. Mayor-Ibarguren et al. [39] reported 83-year-old woman with palpable purpura and serohaematic blisters on lower legs, feet and toes with a history of five days. This patient didn't have COVID-19 symptoms at admission but, she had experienced pharyngeal complaints one-month before from admission. Serologic evaluation revealed IgG and IgM antibodies against SARS-COV-2. Lesions healed in 10 days with systemic steroid treatment. Histopathologic features were depending on lesion type. Palpable purpura was characterized by basal layer necrosis, small vessel injury with fibrinoid necrosis, neutrophilic infiltration vessel walls, leukocytoclasia and erythrocyte extravasation $[38,39]$. Purpuric patches and retiform purpura showed vascular ectasia, thrombi in deep-seated vessels and thrombogenic vasculopathy with perivascular and interstitial neutrophilic infiltration with leukocytoclasia. Extensive necrosis of epidermis, adnexal structures and eccrine coil was also accompanied [34]. Complement deposition were observed in both types of lesions $[34,38]$.

\section{Vesicular Eruption}

Two specific clinical patterns have been reported about vesicular eruptions. Fernandez-Nieto et al. [40] performed a study about vesicular rashes that developed in COVID-19 patients and they observed two main specific eruptions in 24 individuals: diffuse pattern and localized pattern. Diffuse pattern was seen in 18 patients and characterized with widespread polymorphic lesions consisted with 7-8 mm sized papules, vesicles, pustules mainly located on trunk, palms and soles. Localized pattern consisted with monomorphic, 3-4 mm sized vesicles involved more than one region but, primarily trunk. No difference in demographics, clinical features and COVID-19 severity was found between two groups. In total mean age was 45 (range: 19-65) and 18 of them were females. Two patients developed rash before COVID-19 symptoms and three with at the same time of symptoms began. Nineteen patients' lesions appeared after COVID-19 symptoms with a mean latency period of 14 days (4-30). Mean duration of lesions were 10 days (4-22). COVID-19 RNA was searched in four patients' vesicles but, it wasn't presented. Ten patients developed pneumonia and one of them required ICU. Others showed mild disease. Galvan Casas et al. [24] reported 34 patients over 375 suspected and confirmed COVID-19 patients (9\%). Lesions consisted with itching monomorphic vesicles located on trunk and limbs which progressed bigger and diffuse lesions with serohemorrhagic content in several patients. Patients were middle-aged (mean: 45.6; SD: 20) and 19 of them were female (56\%). Five of them developed lesions before and 10 patients after COVID-19 symptoms (15\%). Nineteen at the same time with COVID-19 symptoms. These patients tent to show COVID-19 in moderate severity. Duration of lesions were 10.4 (SD: 9.3) days. Recalcati [21] reported one patient with chickenpox-like vesicles over 84 COVID-19 patients. De Giorgi et al. [22] reported two patients with varicelliform, scattered, vesicles in whom herpes simplex virus (HSV) and varicella-zoster virus (VZV) infections were excluded by PCR analysis. Vesicular lesions due to HSV infection 
were also reported in COVID-19 patients. Hedou et al. [20] reported HSV type 1 activation in intubated patient cared in ICU. Tammaro et al. [41] reported three patients from two hospitals. Two of them had itchy herpetiform vesicles with erythematous halo on trunk. Other patient had numerous vesicles on back. They suggested lesions were caused by agents belonging to Herpesviridae family. Reported histopathologic features were acantholysis, intraepidermal vesicles and ballooned keratinocytes [40].

\section{Mottling}

Only one patient has been reported with mottling. Fifteen-dayold neonate in whom fever, tachycardia (heart rate: 170 beat per minute), tachypnea (respiratory rate: 66 per minute), mild subcostal retractions were accompanied to rash at admission. He was care in ICU because of respiratory distress. He was discharged after six days [42].

\section{Discussion}

In our review we found 34 relevant articles which consisted of 563 COVID-19 patients with cutaneous findings (Table 3). Details of articles and authors can be found in Table 2. The most frequent cutaneous findings were urticarial rashes followed by chilblain-like lesions. Other frequent skin findings were vesicular lesions, erythematous rash, livedo-like lesions and acro-ischemia, respectively. Interestingly, in case series of Galvan Casas et al. [24] the total number of other maculopapular lesions such as perifollicular lesions, pityriasis rosea like lesions, erythema elevatum diutinum like lesions, erythema-multiforme like lesions were higher than all other eruptions.

Urticarial and maculopapular rashes are common lesions and may be related to many different conditions. Most important differential diagnosis is drug eruptions and lots of COVID-19 patients had already taken drugs before rashes appeared. Galvan Casas [24] suggested that these lesions weren't enough for diagnosis but, on the other hand, rash usually onset with COVID-19 symptoms therefore, they might be beneficial for suspecting COVID-19. Although these authors reported that these lesions tent to be developed in more severe patients, De Giorgi et al. [22] didn't observe correlation between these lesions and prognosis [24].

Reports of livedo-like lesions and acro-ischemia are few and reported demographic and clinical features were varied. Although specific data of age were varied and missing in several articles, it seems that lesions were observed in patients around age of 60 $[24,35]$. On the other hand, these lesions might be related with severe disease, requirement of ICU, increased mortality [9,24,34,35]. Galvan Casas et al. [24] reported mortality of these patients as 10 percent [35]. But, however, reports were few. Due to reticulated pattern and cyanosis, vascular involvement was suspected [34,35].
Magro et al. [34] reported patient with altered coagulation markers and complement deposition which might lead to further activation of coagulation in tissues. Reported histopathological feature in two patients revealed thrombosis in deep dermal vessels without obvious vasculitis $[34,35]$. Also, two reported patients with acroischemia showed leg thrombosis [23]. It is not surprising because hypercoagulable state and tendency to clotting is well-known features of COVID-19 disease and can be responsible for this skin manifestations through released cytokines [9,24,34].

Chilblain-like lesions were one of the most argued cutaneous findings of COVID-19 for its nomenclature and association with disease. Due to its acral involvement these lesions were mostly confused with acro-ischemia. Of course, vascular involvement possibly existed but, necrosis was absent. Furthermore, features of these lesions were similar with chilblains. Finally, literature review and suggestion has been made by Piccolo and Bassi [43] who concluded that lesions were different from acro-ischemia and should be called "chilblain-like lesions". Other argued aspect is specifity for COVID-19. In a study of Galvan Casas et al. [24] both confirmed and suspected (couldn't had been confirmed by virologic tests) patients were reported. In their report only $41 \%$ of patients with chilblain-like lesions had got certain diagnosis of COVID-19. As aforementioned before, tele-dermatologic observation of individuals with chilblain-like lesions by Guarneri et al. [23] showed only $26.3 \%$ positivity in PCR analysis of nasopharyngeal swabs. Docampo-Simon et al. [44] prospectively followed 58 individuals with acral lesions during pandemic. Fortytwo of them (72.4\%) had chilblain-like lesions. Thirty-nine of them were tested with PCR and only one of them resulted positive whom lesions regarded as not to be related with COVID-19. de Masson et al. [36] reported 106 individuals with chilblain-like lesions. Only several of them gave positive result for SARS-COV-2. Recalcati et al. [45] reported 11 children and three adults with chilblain-like lesions. Only three of them gave history of cough and fever before three weeks from appearance of rash. Some of these individuals were negative for COVID-19 and others weren't tested. All lesions resolved in 2-4 weeks and no etiologic factors were found. Cordoro et al. [46] reported six adolescents with chilblain-like lesions. Each three of them were clustered in two families. They reported viral upper respiratory infection symptoms 1-2 weeks before onset of rash. All were negative for SARS-COV-2 PCR test. Due to few data and individuals with negative PCR tests, several authors suggested that chilblain-like lesions weren't related with COVID-19 [44]. However, some of these individuals were seen in relatively warmer weathers which was unexpected because nature of chilblains suggests triggering by cold [24,36,43]. Furthermore, clustered cases with chilblain-like lesions into same families were also reported $[24,45,46]$. On the other hand, it seems that this kind 
of lesions tent to be develop in younger patients and later period of COVID-19 which might explain negative PCR results $[9,24,45]$. Chilblain-like lesions seem to be suggestive for COVID-19, seen in younger patients, developed in later periods of disease, and may aid to find out asymptomatic and mild patients.

Petechial and purpuric lesions may be occurred due to vasculitis or thrombogenic vasculopathy [5]. Pathologic features of vasculitis consisted of leukocytoclastic vasculitis and may be caused by immune response against viral antigen accumulation [38,39]. Dominguez-Santas et al. [38] searched for SARS-COV-2 RNA in lesions but, they couldn't find out. They explained that finding with responsible immune complexes didn't contain compact viruses. Although absence of absence of viral RNA in lesions they suggested that reported patient related to COVID-19 and any cutaneous small vessel vasculitis observed during pandemic shouldn't be regarded as idiopathic unless COVID-19 ruled out. Thrombogenic vasculopathy related purpura might be caused by complement activation due to either systemic cytokine escape or direct induction of viral particles seated on lesions [9,30,34]. Magro et al. [34] showed co-localization of SARS-COV-2 spike and envelope proteins with Complement $4 \mathrm{~d}$ and C5b-9 membrane attack complexes on vessels of lungs. They also suggested that focally activated complement system subsequently lead to microvascular injury coagulation and fibrin deposition. They also reported elevated d-dimer levels in serum of their patients. Although reported age and related COVID-19 prognosis were varied in articles, it seems that these kinds of lesions tend to occur in advanced aged patients with coagulation problems and poor prognosis [22,30,39].

Pathologic features of vesicular rash in COVID-19 patients resembled other viral infections and acantholysis seemed to be important mechanism in intra-epidermal blistering. Although FernandezNieto et al. [40] couldn't detect SARS-COV-2 RNA in blister fluid, they pointed that drug usage before appearance of lesions existed in few patients and medical history of patients strongly suggested that vesicles were related with COVID-19. They explained undetected RNA in vesicle contents with false negativity of PCR and absence of standardized methods. However, several authors related their patients' lesions to herpes virus infections, and they suggested that these kinds of lesions weren't specific to COVID-19 [20,41]. Nevertheless, both chicken pox like vesicles and monomorphic scattered lesions were observed in many COVID-19 patients in most of whom drug eruption was unlikely and relationship with disease was strongly suggested [24,40,47]. Therefore, more studies and reports were required to reveal relationship between this kind of lesion type and COVID-19 [41].

In conclusion, reported cutaneous findings in COVID-19 were varied in their clinical appearance. Classification seems to be beneficial to identify these lesions however, different categorizations and nomenclatures exist in articles. Also, association of these lesions with COVID-19 is still speculative and more studies are needed to clarify. Maculopapular and urticarial rash can be seen in other viral exanthems and drug eruptions. But, nevertheless, these conditions were searched, and association was suggested in reports. Livedolike lesions and acro-ischemia may be related hypercoagulable state in COVID-19 patients and also predict poor prognosis. Chilblainlike tend to appear young patients at late stages of mild diseases. Interestingly, these lesions were also reported in clustered normal patients during warm weathers unexpectedly. These patients might had exposed to subtle or asymptomatic disease before admitted to health-care centers. Thus, chilblain-like lesions may be beneficial to catch asymptomatic patients. Petechial lesions seem to be relatively specific to COVID-19 and can occurred due to either true vasculitis or thrombogenic vasculopathy. The latter condition may be associated with severe disease. HSV, VZV and other viral infections should be considered for vesicular lesions that appeared in COVID-19. More articles and studies are needed to determine features of other maculopapular lesions and less frequently reported cutaneous findings such as erythematous rash, mottling, erythema multiformelike lesions and pityriasis rosea like lesions. We observed that different classifications, lack of patient and clinical data and photographs in some articles, scarcity of histopathological investigations. These needed to be updated and reorganized and caused limitations to our review. Other limitations were searching articles in only one database, couldn't access publications with languages other than English and databases for COVID-19. More specific studies, extended case series and reports with histopathological examination are necessary to clarify characteristics, pathogenesis and relations with COVID-19.

\section{Ethics}

Peer-review: Internally peer-reviewed.

\section{Authorship Contributions}

Concept: M.C.K., H.S., Design: M.C.K., H.S., Data Collection or Processing: M.C.K., H.S., Analysis or Interpretation: M.C.K., H.S., Literature Search: M.C.K., H.S., Writing: M.C.K., H.S

Conflict of Interest: No conflict of interest was declared by the authors.

Financial Disclosure: The authors declared that this study received no financial support.

\section{References}

1. Uddin M, Mustafa F, Rizvi TA, Loney T, Suwaidi HA, Al-Marzouqi AHH, Eldin AK, Alsabeeha N, Adrian TE, Stefanini C, Nowotny N, Alsheikh-Ali A, Senok AC. SARS-CoV-2/COVID-19: Viral Genomics, Epidemiology, Vaccines, and Therapeutic Interventions. Viruses 2020;12:526. 
2. Alradhawi M, Shubber N, Sheppard J, Ali Y. Effects of the COVID-19 pandemic on mental well-being amongst individuals in society- A letter to the editor on "The socio-economic implications of the coronavirus and COVID-19 pandemic: A review”. Int J Surg 2020;78:147-148.

3. Ahn DG, Shin HJ, Kim MH, Lee S, Kim HS, Myoung J, Kim BT, Kim SJ. Current Status of Epidemiology, Diagnosis, Therapeutics, and Vaccines for Novel Coronavirus Disease 2019 (COVID-19). J Microbiol Biotechnol 2020;30:313324.

4. Rothan HA, Byrareddy SN. The epidemiology and pathogenesis of coronavirus disease (COVID-19) outbreak. J Autoimmun 2020;109:102433.

5. Wollina U, Karadag AS, Rowland-Payne C, Chiriac A, Lotti T. Cutaneous signs in COVID-19 patients: A review. Dermatol Ther 2020:e13549.

6. Almutairi N, Schwartz RA. Coronavirus Disease-2019 with Dermatologic Manifestations and Implications: An Unfolding Conundrum. Dermatol Ther 2020:e13544.

7. Tang K, Wang Y, Zhang H, Zheng Q, Fang R, Sun Q. Cutaneous manifestations of the Coronavirus Disease 2019 (COVID-19): A brief review. Dermatol Ther 2020:e13528.

8. Ghazal S, Litvinov IV, Aljahani N, Jfri A, Netchiporouk E. Cutaneous Manifestations of Coronavirus Disease 2019 (COVID-19) Infection-What Do We Know So Far? J Cutan Med Surg 2020:1203475420928375.

9. Suchonwanit P, Leerunyakul K, Kositkuljorn C. Diagnostic and prognostic values of cutaneous manifestations in COVID-19. Dermatol Ther 2020:e13650.

10. Sachdeva M, Gianotti R, Shah M, Lucia B, Tosi D, Veraldi S, Ziv M, Leshem E, Dodiuk-Gad RP. Cutaneous manifestations of COVID-19: Report of three cases and a review of literature. J Dermatol Sci 2020;98:75-81.

11. Paolino G, Canti V, Mercuri SR, Rovere Querini P, Candiani M, Pasi F. Diffuse cutaneous manifestation in a new mother with COVID-19 (SARS-Cov-2). Int J Dermatol 2020;10.1111/ijd.14919.

12. Ahouach B, Harant S, Ullmer A, Martres P, Begon E, Blum L, Tess O, Bachmeyer C. Cutaneous lesions in a patient with COVID-19: are they related? $\mathrm{Br} J$ Dermatol 2020;10.1111/bjd.19168.

13. Morey-Olive M, Espiau M, Mercadal-Hally M, Lera-Carballo E, Garcia-Patos V. Cutaneous manifestations in the current pandemic of coronavirus infection disease (COVID 2019). An Pediatr (Engl Ed) 2020;27.

14. Avellana Moreno R, Estela Villa LM, Avellana Moreno V, Estela Villa C, Moreno Aparicio MA, Avellana Fontanella JA. Cutaneous manifestation of COVID-19 in images: a case report. J Eur Acad Dermatol Venereol 2020;24.

15. Putra BE, Adiarto S, Dewayanti SR, Juzar DA. Viral exanthem with "Spins and needles sensation" on extremities of a COVID-19 patient: A self-reported case from an Indonesian medical frontliner. Int J Infect Dis 2020;96:355358

16. Herrero-Moyano M, Capusan TM, Andreu-Barasoain M, Alcantara-Gonzalez J, Ruano-Del Salado M, Sanchez-Largo Uceda ME, Calzado-Villarreal L, Perez-Gonzalez Y. A clinicopathological study of 8 patients with COVID-19 pneumonia and a late-onset exanthema. J Eur Acad Dermatol Venereol 2020;10.1111/jdv.16631.

17. Zengarini C, Orioni G, Cascavilla A, Horna Solera C, Fulgaro C, Misciali C, Patrizi A, Gaspari V. Histological pattern in Covid-19 induced viral rash. J Eur Acad Dermatol Venereol 2020;10.1111/jdv.16569.

18. Amatore F, Macagno N, Mailhe M, Demarez B, Gaudy-Marqueste C, Grob JJ, Raoult D, Brouqui P, Richard MA. SARS-CoV-2 infection presenting as a febrile rash. J Eur Acad Dermatol Venereol 2020:10.1111/jdv.16528.

19. Gianotti R, Veraldi S, Recalcati S, Cusini M, Ghislanzoni M, Boggio F, Fox LP. Cutaneous Clinico-Pathological Findings in three COVID-19-Positive Patients Observed in the Metropolitan Area of Milan, Italy. Acta Derm Venereol 2020;100:adv00124.
20. Hedou M, Carsuzaa F, Chary E, Hainaut E, Cazenave-Roblot F, Masson Regnault M. Comment on "Cutaneous manifestations in COVID-19: a first perspective " by Recalcati S. J Eur Acad Dermatol Venereol 2020;10.1111/ jdv.16519.

21. Recalcati S. Cutaneous manifestations in COVID-19: a first perspective. J Eur Acad Dermatol Venereol 2020;34:e212-e213.

22. De Giorgi V, Recalcati S, Jia Z, Chong W, Ding R, Deng Y, Scarfi F, Venturi F, Trane L, Gori A, Silvestri F, Gao XH, Lotti T. Cutaneous manifestations related to coronavirus disease 2019 (COVID-19): A prospective study from China and Italy. J Am Acad Dermatol 2020;S0190-962230941-962230945.

23. Guarneri C, Venanzi Rullo E, Gallizzi R, Ceccarelli M, Cannavo SP, Nunnari G. Diversity of clinical appearance of cutaneous manifestations in the course of COVID-19. J Eur Acad Dermatol Venereol 2020;10.1111/jdv.16669.

24. Galvan Casas C, Catala A, Carretero Hernandez G, Rodriguez-Jimenez P, Fernandez-Nieto D, Rodriguez-Villa Lario A, Navarro Fernandez I, RuizVillaverde R, Falkenhain-Lopez D, Llamas Velasco M, Garcia-Gavin J, Baniandres O, Gonzalez-Cruz C, Morillas-Lahuerta V, Cubiro X, Figueras Nart I, Selda-Enriquez G, Romani J, Fusta-Novell X, Melian-Olivera A, Roncero Riesco M, Burgos-Blasco P, Sola Ortigosa J, Feito Rodriguez M, Garcia-Doval I. Classification of the cutaneous manifestations of COVID-19: a rapid prospective nationwide consensus study in Spain with 375 cases. $\mathrm{Br}$ J Dermatol 2020;10.1111/bjd.19163.

25. Ehsani AH, Nasimi M, Bigdelo Z. Pityriasis rosea as a cutaneous manifestation of COVID-19 infection. J Eur Acad Dermatol Venereol 2020;10.1111/jdv.16579.

26. Janah H, Zinebi A, Elbenaye J. Atypical erythema multiforme palmar plaques lesions due to Sars-Cov-2. J Eur Acad Dermatol Venereol 2020;10.1111/ jdv.16623.

27. Jimenez-Cauhe J, Ortega-Quijano D, Carretero-Barrio I, Suarez-Valle A, Saceda-Corralo D, Moreno-Garcia Del Real C, Fernandez-Nieto D. Erythema multiforme-like eruption in patients with COVID-19 infection: clinical and histological findings. Clin Exp Dermatol 2020;10.1111/ced.14281.

28. Cepeda-Valdes R, Carrion-Alvarez D, Trejo-Castro A, Hernandez-Torre M, Salas-Alanis J. Cutaneous manifestations in COVID-19: Family cluster of Urticarial Rash. Clin Exp Dermatol 2020;10.1111/ced.14290.

29. Gunawan C, Angela A, Widysanto A. Urticarial eruption in coronavirus disease 2019 infection: a case report in Tangerang, Indonesia. J Eur Acad Dermatol Venereol 2020;10.1111/jdv.16622.

30. Castelnovo L, Capelli F, Tamburello A, Faggioli PM, Mazzone A. Symmetric cutaneous vasculitis in COVID-19 pneumonia. J Eur Acad Dermatol Venereol 2020;10.1111/jdv.16589.

31. van Damme C, Berlingin E, Saussez S, Accaputo O. Acute urticaria with pyrexia as the first manifestations of a COVID-19 infection. J Eur Acad Dermatol Venereol 2020:10.1111/jdv.16523.

32. Henry D, Ackerman M, Sancelme E, Finon A, Esteve E. Urticarial eruption in COVID-19 infection. J Eur Acad Dermatol Venereol 2020:10.1111/jdv.16472.

33. Fernandez-Nieto D, Ortega-Quijano D, Segurado-Miravalles G, PindadoOrtega C, Prieto-Barrios M, Jimenez-Cauhe J. Comment on: Cutaneous manifestations in COVID-19: a first perspective. Safety concerns of clinical images and skin biopsies. J Eur Acad Dermatol Venereol 2020;10.1111/ jdv.16470.

34. Magro C, Mulvey JJ, Berlin D, Nuovo G, Salvatore S, Harp J, Baxter-Stoltzfus A, Laurence J. Complement associated microvascular injury and thrombosis in the pathogenesis of severe COVID-19 infection: A report of five cases. Transl Res 2020;220:1-13.

35. Llamas-Velasco M, Munoz-Hernandez P, Lazaro-Gonzalez J, Reolid-Perez A, Abad-Santamaria B, Fraga J, Dauden-Tello E. Thrombotic occlusive vasculopathy in skin biopsy from a livedoid lesion of a COVID-19 patient. $\mathrm{Br}$ J Dermatol 2020;10.1111/bjd.19222. 
36. de Masson A, Bouaziz JD, Sulimovic L, Cassius C, Jachiet M, Ionescu MA, Rybojad M, Bagot M, Duong TA, Sndv. Chilblains are a common cutaneous finding during the COVID-19 pandemic: a retrospective nationwide study from France. J Am Acad Dermatol 2020;50190-9622(20)30789-30781.

37. Papa A, Salzano AM, Di Dato MT, Varrassi G. Images in Practice: Painful Cutaneous Vasculitis in a SARS-Cov-2 IgG-Positive Child. Pain Ther 2020;1-3.

38. Dominguez-Santas M, Diaz-Guimaraens B, Garcia Abellas P, Moreno-Garcia Del Real C, Burgos-Blasco feminine P, Suarez-Valle A. Cutaneous smallvessel vasculitis associated with novel 2019 coronavirus SARS-CoV-2 infection (COVID-19). J Eur Acad Dermatol Venereol 2020;10.1111/jdv.16663.

39. Mayor-Ibarguren A, Feito-Rodriguez M, Quintana Castanedo L, Ruiz-Bravo E, Montero Vega D, Herranz-Pinto P. Cutaneous small vessel vasculitis secondary to COVID-19 infection: A case report. J Eur Acad Dermatol Venereol 2020;10.1111/jdv.16670.

40. Fernandez-Nieto D, Ortega-Quijano D, Jimenez-Cauhe J, Burgos-Blasco P, de Perosanz-Lobo D, Suarez-Valle A, Cortes-Cuevas JL, Carretero I, Garcia Del Real CM, Fernandez-Guarino M. Clinical and histological characterization of vesicular COVID-19 rashes: A prospective study in a tertiary care hospital. Clin Exp Dermatol 2020:10.1111/ced.14277.

41. Tammaro A, Adebanjo GAR, Parisella FR, Pezzuto A, Rello J. Cutaneous manifestations in COVID-19: the experiences of Barcelona and Rome. J Eur Acad Dermatol Venereol 2020;10.1111/jdv.16530.

42. Kamali Aghdam M, Jafari N, Eftekhari K. Novel coronavirus in a 15-dayold neonate with clinical signs of sepsis, a case report. Infect Dis (Lond) 2020;52:427-429.
43. Piccolo V, Bassi A. Acral findings during the COVID-19 outbreak: Chilblain-like lesions should be preferred to acro-ischemic lesions. J Am Acad Dermatol 2020:50190-9622(20)30946-30944.

44. Docampo-Simon A, Sanchez-Pujol MJ, Juan-Carpena G, Palazon-Cabanes JC, Vergara-De Caso E, Berbegal L, Poveda-Montoyo I, Pastor-Tomas N, MataixDiaz J, Terencio-Alemany C, Martinez-Torres A, Miralles-Botella J, BlanesMartinez M, Gonzalez-Villanueva I, Betlloch-Mas I. Are chilblain-like acral skin lesions really indicative of COVID-19? A prospective study and literature review. J Eur Acad Dermatol Venereol 2020;10.1111/jdv.16665.

45. Recalcati S, Barbagallo T, Frasin LA, Prestinari F, Cogliardi A, Provero MC, Dainese E, Vanzati A, Fantini F. Acral cutaneous lesions in the time of COVID-19. J Eur Acad Dermatol Venereol 2020:10.1111/jdv.16533.

46. Cordoro KM, Reynolds SD, Wattier R, McCalmont TH. Clustered Cases of Acral Perniosis: Clinical Features, Histopathology and Relationship to COVID-19. Pediatr Dermatol 2020;10.1111/pde.14227.

47. Elmas OF, Demirbas A, Ozyurt K, Atasoy M, Tursen U. Cutaneous manifestations of COVID-19: A review of the published literature. Dermatol Ther 2020:10.1111/dth.13696.

48. Estebanez A, Perez-Santiago L, Silva E, Guillen-Climent S, Garcia-Vazquez A, Ramon MD. Cutaneous manifestations in COVID-19: a new contribution. J Eur Acad Dermatol Venereol 2020;10.1111/jdv.16474. 